\title{
VIDAL LORENZO Cristina y Gaspar MUÑOZ COSME (eds), Los grafitos mayas: cuadernos de arquitectura y arqueología maya
}

\section{Claude-François Baudez}

\section{(2) OpenEdition}

\section{Journals}

Édition électronique

URL : http://journals.openedition.org/jsa/11778

DOI : 10.4000/jsa. 11778

ISSN : 1957-7842

Éditeur

Société des américanistes

\section{Édition imprimée}

Date de publication : 5 octobre 2011

Pagination : $324-328$

ISSN : 0037-9174

\section{Référence électronique}

Claude-François Baudez, « VIDAL LoREnzo Cristina y Gaspar muñoz cosme (eds), Los grafitos mayas:

cuadernos de arquitectura y arqueología maya », Journal de la Société des américanistes [En ligne], 97-1 ।

2011, mis en ligne le 10 juillet 2011, consulté le 23 septembre 2020. URL : http://

journals.openedition.org/jsa/11778; DOI : https://doi.org/10.4000/jsa.11778

Ce document a été généré automatiquement le 23 septembre 2020.

(C) Société des Américanistes 


\title{
VIDAL LORENZO Cristina y Gaspar MUÑOZ COSME (eds), Los grafitos mayas: cuadernos de arquitectura $y$ arqueología maya
}

\author{
Claude-François Baudez
}

\section{RÉFÉRENCE}

VIDAL LORENZO Cristina y Gaspar MUÑOZ COSME (eds), Los grafitos mayas: cuadernos de arquitectura y arqueología maya, 2, Universitat politecnica de Valencia/Vicerectorat de cultura, Generalitat valenciana/Conselleria d'educacío, Valencia, 2008, 207 p., bibl., ill. coul., fig., plan, photo, schéma

1 Cet ouvrage collectif est un recueil de contributions à une réunion qui s'est tenue à Valence en décembre 2008. Sa parution, moins d'une année plus tard, est exceptionnellement rapide. L'ouvrage est luxueusement édité sur beau papier, avec de nombreuses photographies en couleur et une mise en pages soignée. Il comprend trois articles généraux ou de synthèse, signés respectivement Karl Herbert Mayer, Dominique Michelet et Miguel Rivera Dorado; des analyses de graffitis dans la microrégion de Rio Bec (Julie Patrois et Philippe Nondédéo), dans les sites de Comalcalco (Miriam Judith Gallegos Gómora et Ricardo Armijo Torres), Chichen Itzá (Ana M. Martín Díaz et Peter J. Schmidt), La Blanca (Cristina Vidal Lorenzo et Gaspar Muñoz Cosme), Nakum et Yaxhá (Jarosław Źrałka et Bernard Hermes). On y trouve également un article sur la conservation et la technique des supports (Begoña Carrascosa Moliner, Montserrat Lastras Pérez et Francisca Lorenzo Mora) et un autre sur l'armement maya à partir des graffitis (Ricardo Torres Marzo). Un graffiti colonial de 1752 à La Blanca, accompagné de documents d'archives sur le signataire (Gaspar Muñoz Cosme, Cristina Vidal Lorenzo et Óscar Haeussler Paredes), clôt la série. 
2 Je partage entièrement l'avis de Mayer, repris par Michelet, que les dessins incisés sur briques de Comalcalco constituent une forme d'expression à part, qui ne peut être assimilée aux graffitis tracés sur le stuc des murs des édifices (surtout) résidentiels. D'abord parce que les briques décorées sont invisibles, puisqu'elles font partie avec d'autres, lisses, de l'appareil des murs et des voûtes des architectures de ce site. Ensuite, parce que chaque brique ornée (non seulement par incision, mais aussi par pastillage, modelage, impression, peinture, pointillé, etc.) ne comporte qu'un seul dessin, ce qui n'est pas le cas des murs à graffitis, où voisinent des dessins indépendants et où s'accumulent des superpositions difficiles à distinguer les unes des autres. Le projet archéologique Comalcalco dispose d'un corpus de 4982 briques décorées, introduites dans la maçonnerie, à plat avec le décor toujours orienté vers le haut; des briques portant le même dessin forment parfois des paires ou des triades. Les motifs sont assez proches des graffitis sur murs des autres sites considérés : des hommes (pas de femmes), des animaux, des architectures (surtout pyramidales), des dessins géométriques. Différentes activités sont représentées (mais pas celles de la «vie quotidienne »), parmi lesquelles un transport de dignitaire en palanquin et des sacrifices par flèches. Les dessins de Comalcalco se distinguent des graffitis d'ailleurs, par quelques glyphes, des chiffres et l'absence de patollis. D'après les auteurs de l'article, les motifs des briques se distribuent différemment dans l'architecture ; ainsi, les animaux et les créatures fantastiques seraient de préférence dans les fondations et les banquettes; les anthropomorphes dans les murs et les piliers; les dessins d'architecture, les scènes complexes et les glyphes, enfin, trouveraient leur place dans les départs de voûte et les frises. Cette distribution différentielle aurait besoin d'être plus précisément définie pour permettre de définir en quoi consistent l'«acte magique » et les « pratiques rituelles », évoqués par Michelet. On souhaiterait connaître la proportion des briques décorées et des briques qui ne le sont pas, la distribution horizontale des motifs dans un même lit, leur répartition verticale dans un mur (analogue aux dépôts cérémoniels stratifiés ?), la possible incidence des orientations cardinales.

3 Si tous les articles font état des techniques de relevé et de conservation, employées dans divers sites, rares sont les chercheurs qui attaquent de front l'important problème des superpositions. En effet, certaines surfaces "graffitées " sont le résultat de cinq superpositions (ou plus ; par exemple : La Blanca fig. 21, p. 111 ; Tikal fig. 32a, 9 in Trik et Kampen 1983 ; Yaxhá fig. 24-25, pp. 152-153) et n'ont aucun sens à être analysées telles quelles. Il est important de distinguer les scènes des unes des autres, à la fois par des moyens techniques (photographiques notamment) et par des analyses stylistiques; ainsi à Tikal, Trik a isolé une partie de jeu de balle - dont le terrain est précédé d'une pyramide avec stèle et autel à son pied - d'un ensemble confus de superpositions, relevé par Kampen (Trik et Kampen 1983, fig. 46). Les motifs qui sont reliés par une même ligne désignant le sol, par exemple, sont supposés appartenir à la même scène ; des figurations à différentes échelles proviennent très probablement de scènes différentes. Il faut être à la fois exigeant et prudent lors de ces analyses sous peine de contresens et de surinterprétations. L'analyse d'une scène de palais à La Blanca (fig. 21, p. 111) est extraite d'un ensemble à plusieurs superpositions; devant un seigneur assis sur son trône, un premier personnage est agenouillé sur une ligne de sol ; à un niveau inférieur, se trouve un autre personnage agenouillé, dessiné à une échelle supérieure et qui flotte, sans être rattaché à une ligne de sol. Il peut s'agir d'un essai antérieur ou d'une copie postérieure. Plus bas, un joueur de trompette ne devrait pas faire partie de 
la même scène, car il tourne le dos au trône, ce qui est peu vraisemblable. Au-dessus du personnage agenouillé de niveau inférieur, plusieurs lignes qui appartiennent sans doute à une scène à deux petits personnages ont été incluses dans la scène de palais (fig. 26) ; en croisant la coiffure de l'agenouillé, elles produisent des hachures que les analystes interprètent comme un filet, que d'aucuns attribuent à la coiffe du «dieu N ».

L'iconographie des graffitis est très limitée: des hommes, des nains, mais pas de femmes; des architectures, mais surtout des pyramides; quelques animaux (serpent, jaguar, oiseaux) de valeur emblématique; des sacrifices par flèches, mais pas de décapitations ni de cardiotomies, ni d'autosacrifices; ni combats, ni captifs. Le personnage assis, que Patrois et Nondédéo interprètent comme un captif, n'en est pas un, car il ne présente aucun signe reconnu de captivité [la ceinture en corde (?) n'en fait pas partie]. Des patollis qui ne sont pas toujours des surfaces de jeu, mais des cosmogrammes, puisque parfois présentés à la verticale (notamment à Nakum et Tikal). La vie quotidienne n'est absolument pas représentée: ni travaux agricoles, ni préparation de nourriture, ni techniques artisanales, ni transactions commerciales.

5 Contrairement à ce que certains auteurs du volume affirment, les graffitis mayas ne sont pas un art populaire, à la fois parce qu'ils sont produits dans des résidences de l'élite par des membres de l'élite, et qu'ils ne sont pas destinés à être vus par le peuple. Ce n'est pas parce que ces dessins sont gauches qu'ils sont "populaires». Leur caractère spontané n'implique pas un intérêt pour la vie quotidienne ou la moindre contestation; les graffitis mayas sont conformistes et les scènes qu'ils s'appliquent à créer sont surtout de caractère politique et rituel. L'immense majorité des graffitis est d'expression maladroite ; ceci ne veut pas dire que les rares dessins réussis sont le fait d'artistes professionnels. Comme partout, il y a des gens qui savent mieux dessiner que d'autres, sans pour autant en faire leur métier.

6 La plupart des graffitis ne font pas partie de scènes, mais sont des motifs indépendants. Le motif le plus courant est un visage humain, de profil, généralement orienté vers la gauche et le plus souvent, inachevé : l'arrière de la tête, la coiffe ou la coiffure ne sont pas indiqués. Ceci nous montre l'importance de la création, même pour figurer le «bonhomme» le plus élémentaire. Ce dernier existe dorénavant sur le mur, quel que soit son degré de ressemblance à des modèles vivants. Les graffitis mayas sont des griffonnages qui n'ont pas d'autre destinataire que leur auteur.

7 Que peut-on tirer de leur étude? Celle de Torres Marzo sur l'armement ne m'a pas convaincu. L'auteur a beaucoup de peine à retrouver dans les dessins ce qu'il sait déjà sur les lances légères et les lances lourdes, et ce ne sont pas les graffitis qui vont l'apprendre; j'ai cherché en vain les masses et les haches; ces dernières, d'après l'iconographie maya classique, ne sont pas des armes de guerre, mais des instruments de sacrifice. Tout en étant sceptique sur la contribution à l'iconographie maya des griffonnages, en considération des limitations de leur pouvoir descriptif, je pense que nous en avons à apprendre sur les représentations mayas, surtout les inconscientes. Prenons l'exemple des dessins "obscènes ", qui n'ont pas été tracés par les habitants des demeures classiques, mais qui sont plutôt fréquents après l'abandon du site, quand ils sont le fait de visiteurs postclassiques. Ces dessins consistent essentiellement en sexes féminins, et l'on ne trouve ni corps féminins, ni seins, ni phallus, contrairement aux graffitis sexuels d'autres cultures.

8 Comme dans les peintures et les bas-reliefs, les visages ne sont pratiquement jamais montrés de face, mais de profil, gauche de préférence. L'étude du style des personnages 
reste à faire. On pourrait, par exemple, profiter de l'extrême simplification de ce graphisme pour relever les parties du corps auxquelles on a accordé plus d'attention, le visage notamment. On pourrait aussi rechercher l'influence de l'art monumental sur les graffitis : ainsi à Tikal, le thème du personnage protecteur du souverain, illustré sur le linteau 2 de la Structure 5C-4 (temple 4 ; Jones et Satterthwaite 1982, fig. 73) se retrouve sur les murs intérieurs des Structures 5D-65 (Trik et Kampen 1983, pp. 66, 71-73) et 5D-91 (ibid., fig. 81). Dans le même site, on pourrait analyser la distribution des deux types de patollis, etc.

9 L'étude des graffitis mayas est en enfance, et un grand avenir lui est promis. Souhaitons que cet ouvrage éveille l'intérêt des chercheurs pour ce domaine si peu exploité et si riche de promesses.

\section{BIBLIOGRAPHIE}

\section{JONES Christopher et Linton SATTERTHWAITE}

1982 The monuments and inscriptions of Tikal : the carved monuments, Tikal Report 33, The University Museum/University of Pennsylvania, Philadelphie.

TRIK Helen et Michael E. KAMPEN

1983 The graffiti of Tikal, Tikal Report 31, The University Museum/University of Pennsylvania, Philadelphie.

\section{AUTEURS}

\section{CLAUDE-FRANÇOIS BAUDEZ}

Directeur de recherche honoraire, CNRS 